\title{
Lesion-Specific Language Network Alterations in Temporal Lobe Epilepsy
}

\author{
(D) O. Foesleitner, (D) K.-H. Nenning, (DL. Bartha-Doering, (DC. Baumgartner, (DE. Pataraia, (DD. Moser, (D) M. Schwarz, \\ (D) V. Schmidbauer, (D).A. Hainfellner, (D). Czech, (D). Dorfer, (D) G. Langs, (DD. Prayer, (D). Bonelli, and (D) Kasprian
}

\begin{abstract}
BACKGROUND AND PURPOSE: Temporal lobe epilepsy, structural or nonlesional, may negatively affect language function. However, little is known about the lesion-specific influence on language networks. We hypothesized that different epileptogenic lesions are related to distinct alterations in the functional language connectome detected by fMRI.
\end{abstract}

MATERIALS AND METHODS: One hundred one patients with epilepsy due to mesiotemporal sclerosis (21 left, 22 right), low-grade mesiotemporal tumors (12 left), or nonlesional temporal lobe epilepsy (22 left, 24 right) and 22 healthy subjects performed $3 T$ taskbased language fMRI. Task-based activation maps (laterality indices) and functional connectivity analysis (global and connectivity strengths between language areas) were correlated with language scores.

RESULTS: Laterality indices based on fMRI activation maps failed to discriminate among patient groups. Functional connectivity analysis revealed the most extended language network alterations in left mesiotemporal sclerosis (involving the left temporal pole, left inferior frontal gyrus, and bilateral premotor areas). The other patient groups showed less extended but also predominantly ipsilesional network changes compared with healthy controls. Left-to-right hippocampal connectivity strength correlated positively with naming function $(P=.01)$, and connectivity strength between the left Wernicke area and the left hippocampus was linked to verbal fluency scores $(P=.01)$ across all groups.

CONCLUSIONS: Different pathologies underlying temporal lobe epilepsy are related to distinct alterations of the functional language connectome visualized by fMRI functional connectivity analysis. Network analysis allows new insights into language organization and provides possible imaging biomarkers for language function. These imaging findings emphasize the importance of a personalized treatment strategy in patients with epilepsy.

ABBREVIATIONS: $\mathrm{FC}=$ functional connectivity; $\mathrm{HC}=$ hippocampus; $\mathrm{LI}=$ laterality index; $\mathrm{MTS}=$ mesiotemporal sclerosis; $\mathrm{nl-TLE}=$ nonlesional TLE; $\mathrm{TLE}=$ temporal lobe epilepsy

$\mathrm{T}$ emporal lobe epilepsy (TLE) can be triggered by various underlying pathologies with mesiotemporal sclerosis (MTS) and low-grade tumors being the most frequent. ${ }^{1}$ In some cases, no structural alteration can be found on MR imaging, which is termed nonlesional (nl-TLE). Despite sharing the same anatomic

Received June 17, 2019; accepted after revision October 21.

From the Departments of Biomedical Imaging and Image-Guided Therapy (O.F., K.-H.N., M.S., V.S., G.L., D.P., G.K.), Neurology (E.P., D.M., S.B.), Pediatrics and Adolescent Medicine (L.B.-D.), Institute of Neurology (J.A.H.), and Neurosurgery (T.C., C.D.), Medical University of Vienna, Vienna, Austria; and General Hospital Hietzing with Neurological Center Rosenhuegel (C.B.), Vienna, Austria.

Please address correspondence to Gregor Kasprian, MD, Department of Biomedical Imaging and Image-Guided Therapy, Medical University of Vienna, Waehringer Gürtel 18-20, 1090 Vienna, Austria; e-mail gregor.kasprian@meduniwien.ac.at

Indicates article with supplemental on-line appendix and tables.

Indicates article with supplemental on-line photos.

http://dx.doi.org/10.3174/ajnr.A6350 location and clinical picture, these conditions differ in clinical and cognitive outcomes and thus require different treatment strategies. $^{2,3}$

Progressive cognitive deficits are common comorbidities in patients with epilepsy. ${ }^{4}$ One-third of patients with TLE have impaired language function, which may further deteriorate after epilepsy surgery. ${ }^{2,5}$ Imaging tools that assess language organization preoperatively are therefore essential to optimize treatment decisions.

Task-based fMRI is a well-established clinical tool for noninvasive presurgical language mapping and shows good concordance with direct cortical stimulation. ${ }^{6,7}$ However, language processing has network properties, ${ }^{8}$ which cannot be fully captured by fMRI activation maps alone. Functional connectivity (FC) analysis uses fMRI data to investigate brain networks ${ }^{9,10}$ and thus opens new perspectives in our understanding of language function. 


\begin{tabular}{|c|c|c|c|c|c|c|c|c|c|c|c|c|}
\hline & \multicolumn{2}{|c|}{ Controls } & \multicolumn{2}{|c|}{ Left nl-TLE } & \multicolumn{2}{|c|}{ Right nl-TLE } & \multicolumn{2}{|c|}{ Left MTS } & \multicolumn{2}{|c|}{ Right MTS } & \multicolumn{2}{|c|}{ Left Tumor } \\
\hline & No. & $\begin{array}{l}\text { Median } \\
\text { (Range) }\end{array}$ & No. & $\begin{array}{l}\text { Median } \\
\text { (Range) }\end{array}$ & No. & $\begin{array}{l}\text { Median } \\
\text { (Range) }\end{array}$ & No. & $\begin{array}{l}\text { Median } \\
\text { (Range) }\end{array}$ & No. & $\begin{array}{l}\text { Median } \\
\text { (Range) }\end{array}$ & No. & $\begin{array}{l}\text { Median } \\
\text { (Range) }\end{array}$ \\
\hline \multicolumn{13}{|l|}{ Sex } \\
\hline Male & 14 & & 14 & & 10 & & 10 & & 16 & & 6 & \\
\hline Female & 8 & & 8 & & 14 & & 11 & & 6 & & 6 & \\
\hline \multicolumn{13}{|l|}{ Handedness } \\
\hline Right & 21 & & 15 & & 17 & & 20 & & 17 & & 10 & \\
\hline Left & 1 & & 6 & & 5 & & 1 & & 4 & & 2 & \\
\hline $\begin{array}{l}\text { Age at fMRI } \\
\text { imaging (yr) }\end{array}$ & & $34(23-52)$ & & $36(21-55)$ & & $3(18-60)$ & & $44(17-64)$ & & 39 (19-52) & & $29(18-54)$ \\
\hline $\begin{array}{l}\text { Age at seizure } \\
\text { onset (yr) }\end{array}$ & & NA & & $23(4-54)$ & & $21(1-35)$ & & 17 (1-41) & & $19(1-42)$ & & $25(12-46)$ \\
\hline $\begin{array}{l}\text { Duration of } \\
\text { epilepsy (yr) }\end{array}$ & & NA & & $9(1-42)$ & & $10(1-43)$ & & $21(3-48)^{a}$ & & $14(1-44)$ & & $6(1-29)^{a}$ \\
\hline
\end{tabular}

Note:-NA indicates not applicable.

${ }^{a}$ Significant difference (post hoc Tukey test, $P=.04$ ).

In comparison with task-based fMRI, FC may be more sensitive to lesion-specific alterations of functional representations and/or network changes. In current scientific and clinical imaging practice, there is a common bias toward the anatomic location and a certain indifference regarding the etiology and type of lesion causing TLE. ${ }^{11,12}$ This study aimed to provide a deeper understanding of the relation among the etiology of epileptogenic lesions, the resulting language network alterations, and cognitive function.

We tested the following major hypotheses: 1) The underlying pathology causing TLE influences the organization of language function, and 2) FC analysis provides additional information compared with standard fMRI activation analysis.

\section{MATERIALS AND METHODS}

The study was approved by the local ethics committee (Medical University of Vienna, Austria; EK 1883/2016) and was conducted in accordance with the Declaration of Helsinki of 1975. All participants gave informed consent.

\section{Participants}

A flow chart listing inclusion and exclusion criteria for patient selection is provided in On-line Fig 1. One hundred one patients with TLE due to MTS (21 left, 22 right), with low-grade epilepsyassociated tumors (12 left) or without structural MR imaging alteration (22 left, 24 right), were included into this retrospective study (demographics are listed in the Table). All patients had task-based language fMRI examinations as part of their presurgical evaluation between March 2011 and June 2018. Clinical data were retrieved from the hospital information system or directly from the physician in charge. Additionally, 22 healthy subjects with no history of neurologic or psychiatric disease, no clinical evidence of neurologic dysfunction, and no structural alteration on MR imaging were scanned with the same imaging protocol as patients.

Diagnosis of unilateral TLE was based on typical seizure semiology and prolonged ictal and interictal video-electroencephalography interpreted by epileptologists. Diagnosis of MTS and nonexistence of other pathologies were confirmed by a trained neuroradiologist with 6 years of experience in epilepsy imaging.
Cases of nl-TLE were selected on the basis of no structural lesion on a $3 \mathrm{~T}$ scan with a dedicated epilepsy protocol, which included sagittal isotropic FLAIR and T1WI, axial DWI and SWI, and coronal slices perpendicular to the long axis of the hippocampus on T2WI and inversion recovery. In cases of tumor, additional contrast-enhanced T1WI was acquired.

Low-grade TLE-associated brain tumors were included if patients fulfilled the following criteria: substantial involvement of the mesiotemporal lobe without any extratemporal impact, MR imaging features typical of a low-grade lesion, and little or no mass effect/perifocal edema (On-line Fig 2). Twelve patients with left-sided tumors fulfilled these criteria (On-line Fig 1). Because only 5 patients with right-sided lesions were eligible, they were excluded from further analysis. Six of the patients with left tumors proceeded to surgery, with histopathology defining an astrocytoma II in 2 cases, a composite/dysembryoplastic neuroepithelial tumor I in 2 cases, and a ganglioglioma $\mathrm{I}$ in 2 cases (grading according to the World Health Organization brain tumor classification system, Version 2007 or 2016, depending on time of reporting ${ }^{13,14}$ ).

Epilepsy surgery was performed on $32 / 43$ patients with MTS (15 left, 17 right), confirming hippocampal sclerosis in 31 cases and revealing an irregular neuronal architecture of the hippocampus (HC) in 1 case, reported as a mild malformation of cortical development. Nineteen of 46 patients with nl-TLE (7 left, 12 right) underwent partial temporal lobe resection with histopathologic work-up finding a focal cortical dysplasia in 11 cases, mild MTS in 4, gliosis in 2, and no abnormal findings in 2.

Neuropsychological assessment as part of presurgical evaluation was performed by 2 experienced neuropsychologists and was retrieved from the hospital information system. We focused on language scores-that is, naming ability assessed with the Boston Naming Test ${ }^{15}$ and verbal fluency assessed with the Regensburger Verbal Fluency Test. ${ }^{16}$ Because for many patients, medical reports did not provide absolute test scores, performance was dichotomized into "below average" and "average or above average."

\section{fMRI Acquisition}

fMRI was performed on the same Achieva 3T scanner (Phillips Healthcare, Best, the Netherlands) in all subjects, using a gradient- 
echo EPI sequence (section thickness, $1.8 \times 1.8 \times 4 \mathrm{~mm}$; TE, $35 \mathrm{~ms}$; TR, $3000 \mathrm{~ms}$; flip angle, $90^{\circ}$; matrix, $128 \times 128$; 32 contiguous slices with no interslice gap; ascending section acquisition; bandwidth, $2280 \mathrm{~Hz} / \mathrm{Px}$ ). Additionally, 1- $\mathrm{mm}$ isotropic T1WI was acquired for anatomic coregistration. Every participant performed validated language tasks in 1-5 runs (verb generation and/or a semantic task; for task descriptions and distributions, see the Online Appendix and On-line Table 1). The paradigms were all constructed in block design with 5 minutes per run, resulting in 100 measured time points per run. A training run before scanning and on-line processing ensured correct in-scanner performance.

\section{Activation Analysis}

First, fMRI data were converted from DICOM format to Neuroimaging Informatics Technology Initiative format (https:// nifti.nimh.nih.gov/) using SPM 12 software (http://www.fil.ion. ucl.ac.uk/spm/software/spm12). ${ }^{17}$ Standard preprocessing was applied-that is, motion correction, coregistration of functionalto-anatomic images, direct spatial normalization to Montreal Neurological Institution space, and smoothing (full width at half maximum, $8 \mathrm{~mm}$ ). First-level analysis was then performed according to the general linear model. Language-related activations were visually checked using SPM 12 ( $P$ uncorrected $<$ .001). Scans were excluded from further analysis if no significant activations were seen in classic language areas (ie, in the inferior frontal gyrus and/or superior temporal gyrus bilaterally; On-line Fig 3). In second-level analysis, task-related activations were contrasted between patient and control groups for each task separately ( $P$ family-wise error-corrected $<.05$ ).

Additionally, a laterality index (LI) was calculated from the individual activation maps for each run using a bootstrap approach as recommended by Wilke and Lidzba. ${ }^{18}$ We used the same ROIs for further FC analysis, with language network ROIs defined as the triangular and opercular parts of the inferior frontal gyrus for the Broca area and a slightly extended region of the posterior superior temporal gyrus for the Wernicke area as well as their right-hemispheric homologs. This parcellation is based on an ICA analysis of 497 subjects from the Human Connectome Project data base and integrated in the CONN toolbox (https:// web.conn-toolbox.org/). ${ }^{19}$ In cases of $>1$ run per task, a mean LI was calculated for each ROI and each task separately. Moreover, per subject an average LI for both Broca and Wernicke ROIs per task, as well as an average LI for both tasks per Broca and Wernicke ROIs, and finally, an average LI for both tasks and both Broca and Wernicke ROIs were calculated. This ultimately resulted in 9 group comparisons for the LI, allowing a multi-layered analysis of lateralization scores. Left-sided language lateralization was defined as LI $\geq 0.2$; atypical LI was accordingly set at $\mathrm{LI}<0.2$, as is common in the literature. ${ }^{20}$

\section{Functional Connectivity Analysis}

FC was analyzed using the CONN toolbox, Version 17.f running on SPM12. ${ }^{19}$ Preprocessing of anatomic and functional data was performed using default settings (functional realignment and unwarping, centering to $[0,0,0]$ coordinates, section-timing correction, artifact detection tools-based outlier detection, direct segmentation and Montreal Neurological Institute normalization, and smoothing with an 8-mm Gaussian kernel). Denoising included GM and WM signal, CSF signal, realignment, and scrubbing parameters. Task design and first-derivative task regression were also included in the denoising step to prevent coactivations being erroneously summarized as a network. ${ }^{21}$ By default, the CONN toolbox uses a principal component analysis approach to reduce motion-related artifacts. ${ }^{22}$ We included all acquired time points ( $n=100$ per run) in our FC analysis in order to not introduce artificial fluctuations into the frequency spectrum by cutting and concatenating task and resting blocks. ${ }^{23}$ Moreover, this approach should maximize the available number of time points, which is known to critically influence the reliability of connectivity measures. ${ }^{24}$ After regression, a high-pass filter (0.008-infinity) was applied for denoising instead of the default bandpass filter for resting-state data, which aimed to keep potentially task-relevant low-frequency fluctuations. ${ }^{25}$ Then, bivariate correlation was calculated for the 103 supratentorial atlases and for 4 (language) network ROIs defined by anatomic parcellation atlases included in the CONN toolbox (FSL Harvard-Oxford atlas; http://neuro.debian.net/ pkgs/fsl-harvard-oxford-atlases.html). The language ROIs were congruent to the ones used in the LI analysis (On-line Fig 3). This feature resulted in Fisher $z$-transformed correlation coefficients for each connection.

First, we analyzed the global language network changes-that is, group differences in the FC strength between the 4 languagenetwork ROIs and 97 anatomic ROIs (ie, disregarding the left and right triangular and opercular inferior frontal gyri and posterior superior temporal gyrus to avoid autocorrelation). For this analysis, a nonparametric approach (network-based analysis) was chosen with a combination of an ROI-to-ROI connection-correction $(P$ uncorrected $<.01)$ and a seed corrected by size $(P$ false discovery rate-corrected $<.05) .{ }^{26}$ Second, changes in the FC between the ipsilesional $\mathrm{HC}$ and the 103 anatomic ROIs at $P$ false discovery rate-corrected (analysis-level correction) $<.05$ were specifically assessed. Patient groups were each contrasted to the healthy control group on the basis of a standard unpaired $t$ test. Finally, FC strength among the 4 language ROIs was analyzed regarding group-specific differences (ANOVA).

\section{Statistical Analysis of Clinical and Imaging Parameters}

We tested group differences regarding sex, handedness, age at fMRI scan, age at first seizure, epilepsy duration, naming, and verbal fluency performance. These clinical values were correlated with LI values (9 values as described above) and FC values (Fisher $z$-transform) between the language ROIs and the left or right HC. Furthermore, motion parameters (mean and maximum motion) were tested for significant group differences to rule out motiondriven false-positive results. Independent $t$ tests or ANOVA was used for continuous variables with the Tukey test for post hoc analysis and $\chi^{2}$ tests for categoric data (SPSS Statistics for Macintosh, Version 25.0, released 2017; IBM, Armonk, New York). Visual inspection of histograms determined the Gaussian distribution of metric variables and, subsequently, selection of parametric (Pearson) or nonparametric (Spearman) correlation tests. Significance was set at $\alpha=.05$ if not indicated otherwise. 


\section{RESULTS}

\section{Clinical Parameters}

There were no significant group differences regarding sex $(P=.43)$, handedness $(P=.2)$, age at fMRI scan $(P=.78)$, or age at first seizure $(P=.2)$. In 4 patients, handedness was not known. The median duration of epilepsy was different between patients with left tumor and left MTS (6 versus 21 years, $P=.04$, post hoc Tukey test). Demographics are outlined in the Table.

Neuropsychological testing was performed in 68/101 patients (67.3\%). Forty-three patients performed well (63.2\%), and 25, below average $(36.8 \%)$ on the verbal fluency test. The naming test resulted in below-average scores in 47/101 (69.1\%) and good scores in 21 patients $(30.9 \%)$. There was a strong correlation among test scores in the different tests $(P=.001)$, while there were no significant differences in verbal fluency or naming scores between groups $(P=.6$ and $P=.67$, respectively).

\section{fMRI Quality Assessment}

From the subjects included, 27/279 fMRI runs had to be excluded due to missing language-related activations, leaving $252 \mathrm{fMRI}$ scans for further analysis (On-line Table 1). There were no significant group differences in mean or maximum interscan movement; hence, group differences in activation and FC analyses are unlikely to be driven by motion artifacts (On-line Fig 4).

\section{fMRI Activation Analysis and Language LI}

There was no significant group difference in activation maps between patient groups and controls ( $P \leq .05$ family-wise errorcorrected for multiple comparisons). Group-level activation maps are shown in On-line Figs 5 and 6.

Calculation of laterality indices did not result in significant differences in any group (ANOVA, listed in On-line Tables 2 and 3). The mean LI for the verb-generation task was left-lateralized in all groups (mean ranging from $0.3 \pm 0.4$ in left nl-TLE to $0.57 \pm 0.29$ in right MTS, Fig 1). The semantic task achieved a slightly lower but still left-lateralized mean LI (ranging from $0.25 \pm 0.41$ in left nl-TLE to $0.53 \pm 0.19$ in left tumor, Fig 1).

Of the 19 left-handed subjects, only 1 patient with left nl-TLE had an atypical LI considering both Broca and Wernicke ROIs; 1 patient with left nl-TLE showed a bilateral language dominance, while the other 17 had a typical LI. Right-handed participants showed a typical LI considering both frontal and temporal language ROIs in 100 cases, bilateral language distribution in 19 cases, and an atypical LI in 4 cases. Handedness was not associated with language lateralization $(P=.5)$.

\section{Functional Connectivity Analysis}

Distinct patterns of FC alterations could be found in the comparison between the control group and each patient group (Fig 2). Most widespread changes were seen in left and right MTS; less significant network alterations, in left and right nl-TLE; and even less, in patients with left tumor. In all except patients with right nl-TLE and left tumor, FC from frontal language areas showed impaired connectivity strength. Temporal language regions were affected in all patient groups (for anterior, left and right projections see On-line Figs 7-11). FC values among the 4 language network ROIs alone did not show significant group differences, nor did direct comparisons among patient groups.

Regarding ipsilesional hippocampal network changes, only patients with left and right MTS showed significant FC changes between the ipsilesional HC and all other ROIs (Fig 3). In both cases, the superior frontal gyrus was bilaterally affected. In left MTS, visual areas and the right temporal pole also showed weaker FC to the ipsilesional HC. In right MTS, both frontal poles as well as the left temporal and right parietal areas were affected.

\section{Correlation of Clinical and Imaging Parameters}

Right- or left-hemispheric TLE was associated with the language LI in the verb-generation task in the Broca area and with the LI in the verb-generation task in both Broca and Wernicke areas, regardless of the underlying pathology $(P=.01$ and $P=.008$, respectively), indicating a more atypical language lateralization in left-sided TLE. Similarly, left seizure onset showed a negative correlation with interhippocampal connectivity $(P=.01)$ and a positive correlation with FC between the right $\mathrm{HC}$ and left Wernicke area $(P=.002)$ across all patients. Boxplots are shown in On-line Fig 12.

Epilepsy duration was negatively correlated with the language LI in the semantic task in Broca and Wernicke areas across all patients $(P<.02)$. The longer the epilepsy duration, the more atypical was the LI (On-line Fig 13). Moreover, epilepsy duration showed a negative correlation with FC between the right $\mathrm{HC}$ and right Wernicke homolog $(P<.02)$ and with $\mathrm{FC}$ between the right $\mathrm{HC}$ and left Broca area $(P<.04)$ across all patients. The longer the epilepsy duration, the weaker were these connections.

Verbal fluency was negatively correlated with FC between the right Broca and Wernicke homologs $(P<.03)$ across all patients (On-line Fig 14). Naming performance showed a positive correlation with FC between the right $\mathrm{HC}$ and left Broca area $(P<.02)$ across all patients (On-line Fig 14). There was no significant correlation between any LI and performance in verbal fluency or naming.

\section{DISCUSSION Key Findings}

This study evaluated the impact of different temporal lobe pathologies, grossly involving the same anatomic locations on language function, cortical representations, and networks. While fMRI activation analysis failed to find significant group differences in cortical language representations, FC analysis detected lesionspecific footprints of language connectome changes. Among all analyzed pathologies, patients with left and right MTS showed the most widespread, bilateral language network alterations. Patients with nl-TLE mainly showed FC decreases in temporal language regions, whereas patients with tumor showed the smallest language network alterations. Moreover, changes in the strength of certain connections correlated with deficits in specific language domains, independent of the underlying lesion. A normal score in verbal fluency was associated with less right hemispheric language connectivity, and naming ability correlated with FC between the right $\mathrm{HC}$ and left Broca area. 


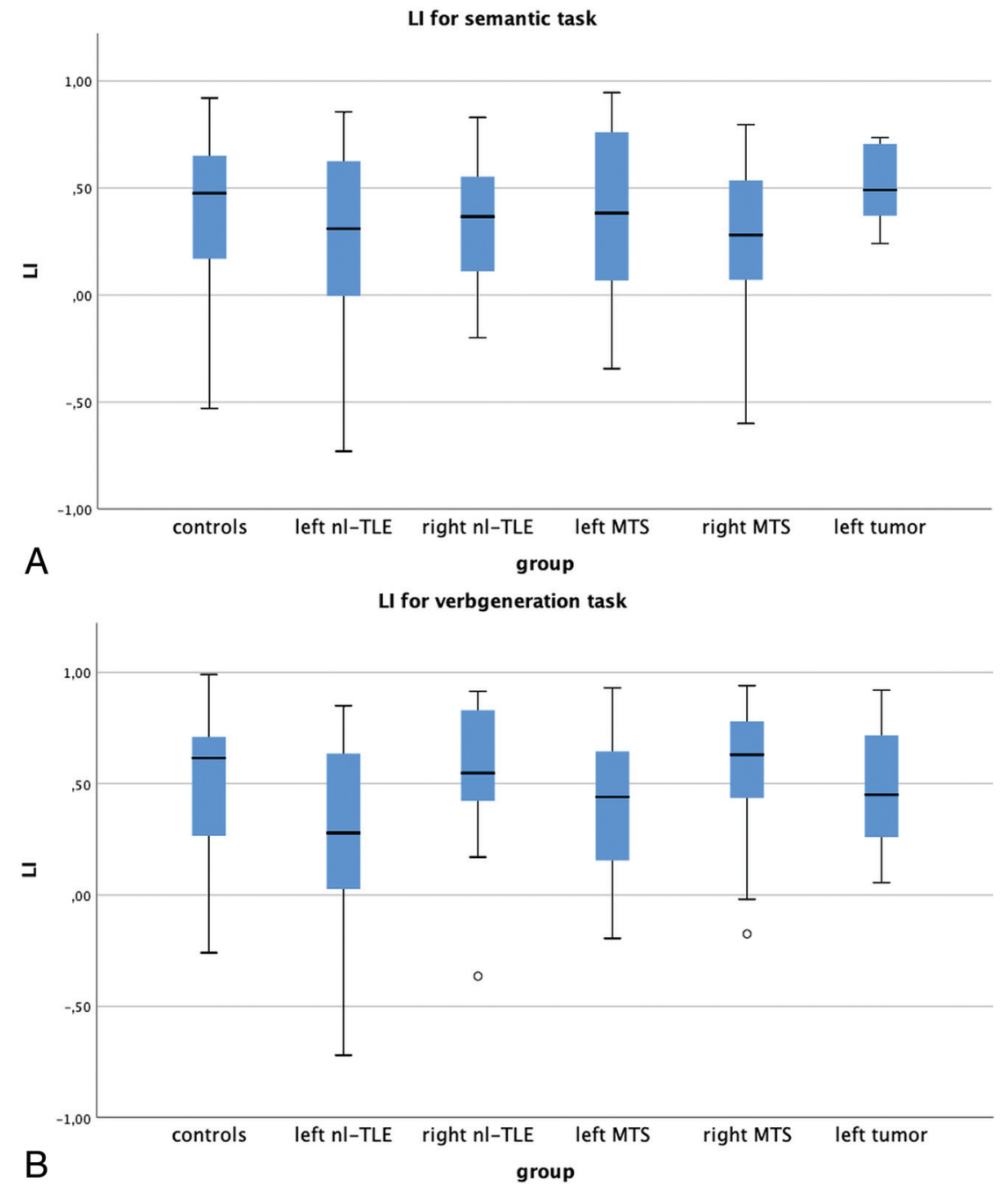

FIG 1. Group-wise boxplot of laterality indices for the verb-generation and semantic tasks. The mean LI was left-lateralized in every group, with no significant group differences at $\alpha=.05$.

graph theory and resting-state data, Vaughan et $\mathrm{al}^{33}$ found a functional segregation of the sclerotic $\mathrm{HC}$ and decreased FC to the frontal lobe. In our cohort, exclusively, the patients with MTS showed significant changes in FC of the ipsilesional HC, including premotor areas and the contralateral temporal pole. In left MTS, left visual areas were also affected, while right MTS showed disrupted FC to right parietal regions. Most interesting, hippocampal language connectivity was minimally affected in nl-TLE and temporal lobe tumors.

\section{Long-Term Epilepsy-Associated Tumors}

Long-term epilepsy-associated tumors are characterized by slow cell growth, rare malignant transformation, young age at epilepsy onset, and predominance for the temporal lobe (ie, proximity to the eloquent cortex). ${ }^{34,35}$ Besides oncologic control, seizure freedom is the main therapeutic goal. In these tumor entities, neuronal dysfunction seems to be less prominent, and functional reorganization, less exclusive compared with other epileptogenic pathologies. ${ }^{36,37}$ Reasons might be their slow growth and young age of onset. ${ }^{38}$ Our findings are in concordance with the existing literature because functional imaging changes in our cohort of left TLE-associated tumors were the least severe. How-

\section{Mesiotemporal Sclerosis}

Histopathologic hallmarks of MTS are segmental loss of pyramidal cells accompanied by astrogliosis. ${ }^{27}$ Despite MTS being a focal lesion, widespread structural alterations such as diffuse GM and WM atrophy were detected in MTS. ${ }^{28,29}$ Similarly, distinct functional changes were visible far beyond the seizure-onset zone. ${ }^{30,31}$ This finding is in line with ours in patients with MTS, who showed the most widespread language network of all patient groups, with alterations involving both hemispheres. The median epilepsy duration was higher in patients with left MTS than in those with left tumors, a finding that certainly influenced our results. However, there was no significant difference compared with the other patient groups and none at all compared with patients with right MTS.

The HC is not considered a "classic" language region, but there is evidence that it makes a pivotal contribution to language function, even more than harvesting verbal memory. ${ }^{32}$ Using resting-state $\mathrm{fMRI}$, de Campos et $\mathrm{al}^{31}$ showed the ipsilesional $\mathrm{HC}$ as the "main hub of alterations" with widespread connectivity reductions in left and right MTS and, specifically, impaired connectivity of the visuo-spatial network in left MTS. Using ever, our data did not show compensatory stronger FC in other brain regions, which could be too subtle for the given sample size of 12 patients.

\section{Nonlesional TLE}

With improved structural imaging (ie, 3T scanners and beyond, dedicated epilepsy protocols), MR imaging negative for TLE is increasingly seen as a distinct entity rather than a subtle form of MTS. ${ }^{39}$ This is suggested by studies using advanced MR imaging techniques, both structural and functional. ${ }^{29,33}$ For instance, in a graph-theory study, nl-TLE showed impaired connectivity in the ipsilateral neocortical but not medial temporal lobe, contrary to MTS with severe affection of the HC. ${ }^{33}$ Our results add to this because the extent of changes in the functional language network in our patients with nl-TLE was significantly less numerous and widespread than in MTS, possibly reflecting the severity of the impact of the pathology on the language network. Clinically, cognitive function is much less frequently impaired in patients with nl-TLE than in those with MTS, which, as a downside, puts them at higher risk of postoperative decline. ${ }^{3}$ 


\section{Language Laterality}

Language is one of the most critical cognitive functions in TLE because it is impaired in every third patient and may further deteriorate after epilepsy surgery. ${ }^{2,5}$ In the general population,

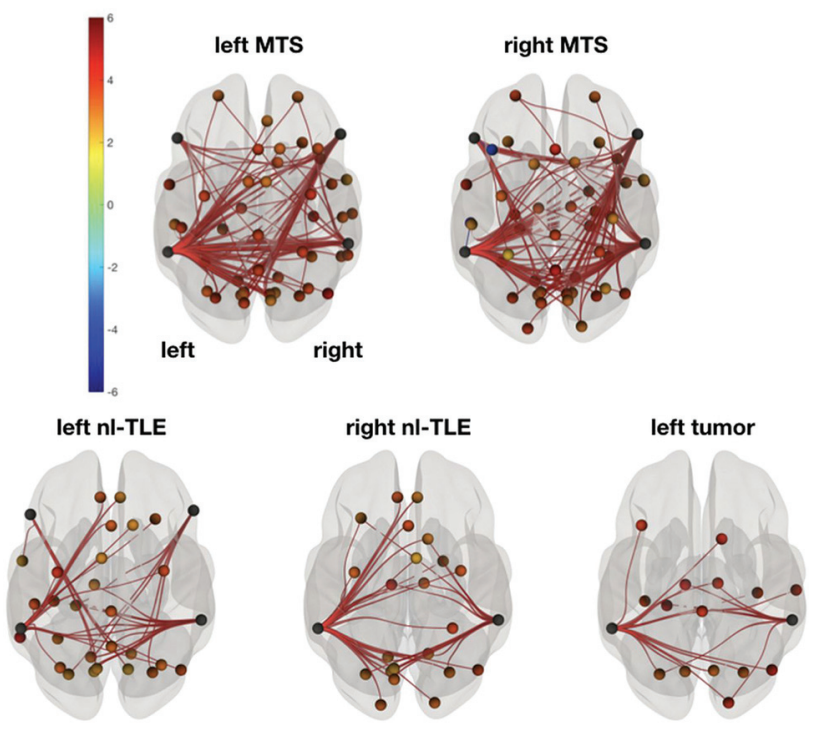

FIG 2. FC contrast between control and patient groups. Left and right MTS was associated with the most widespread, bilateral FC decreases compared with controls. Changes in both frontal and temporal language ROls were found in left nl-TLE, while right nl-TLE and the left tumor groups showed decreases in only temporal language regions. Superior view in neurologic convention. Network based statistics, connection-corrected at $P<.01$ and seed-corrected at $P$ false discovery rate $<.05$; seeds: 4 language network ROls; target ROls: supratentorial brain. Color bar shows $t$ values $(-6 ; 6)$, warm colors meaning decreased, cold colors increased FC strength (controls $>$ patients). language function is mostly left-hemisphere dominant. Especially, left-sided pathologies such as left TLE are associated with a higher prevalence of atypical (ie, bilateral or even rightdominant) language distribution. ${ }^{40}$ Not surprisingly, left seizure onset was linked to an atypical language lateralization, regardless of the underlying pathology in our patients. Handedness is frequently seen as a predictive marker for language lateralization. However, it seems that this assumption is mainly driven by a minority of subjects ( $<1 \%$ of the general population) with strongly atypical language dominance who also have a strong preference for their left hand. ${ }^{41}$ Yet, left-handedness alone is not predictive of language dominance, which also applied to our participants (see "fMRI Quality Assessment").

\section{Activation-versus-FC Analysis}

According to the current neurobiologic concept, language is built on multiple bilateral brain regions. ${ }^{8}$ The current clinical practice of presurgical fMRI is based on task-based activation analyses. While these are adequate to detect task-associated brain regions, they do not have the potential to provide information about the communication among these functional areas. Previous work showed that FC analysis can detect more subtle differences than standard fMRI analysis on a group level. ${ }^{42}$ In 2006, Briellmann et $\mathrm{al}^{43}$ did not find pathology-related differences in standard fMRI laterality indices between MTS and neuroglial tumors. In a small cohort of patients with TLE with mixed etiologies, Pravata et $\mathrm{al}^{42}$ found a correlation between left-intrahemispheric FC and the verbal intelligence quotient in left TLE, but no such association in the task-based LI. Our findings are in line with these studies because only FC analysis found group-specific differences and a significant association between imaging and clinical parameters.
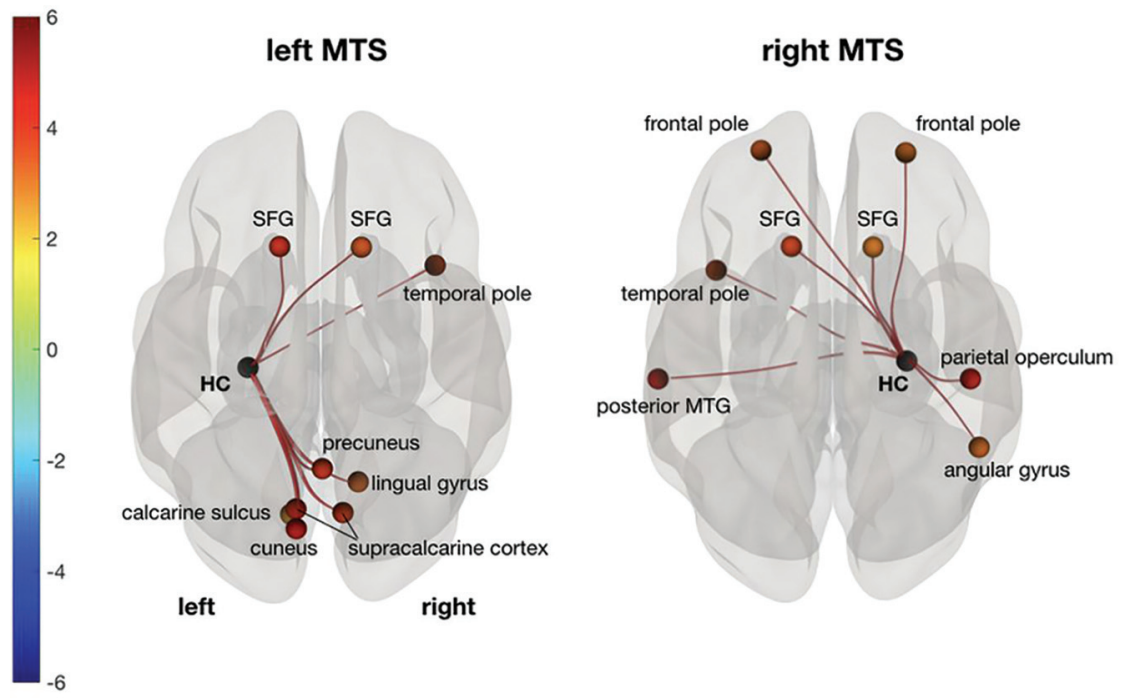

FIG 3. $F C$ changes between the ipsilesional $\mathrm{HC}$ and all other supratentorial ROIs show weaker connectivity to the superior frontal gyrus (SFG) in both patients with left and right MTS compared with controls. In left MTS, visual areas and the right temporal pole are also affected. In right MTS, left temporal, right parietal, and bilateral frontal areas show significant FC decreases. Superior view in neurologic convention. $P$ false discovery rate-corrected $<.05$. Color bar shows $t$ values $(-6 ; 6)$, warm colors meaning decreased, cold colors increased FC strength (controls $>$ patients). MTG indicates middle temporal gyrus.

\section{Task-Based FC}

Most FC analyses are based on spontaneous fluctuations known as restingstate fMRI (ie, subjects being at wakeful rest inside the scanner) reliably revealing major functional networks (eg, sensorimotor or language). However, task-based fMRI enhances specific networks while decreasing others, ${ }^{44}$ and the findings remain different from resting-state data, even with sophisticated postprocessing such as task-regression or concatenation of resting blocks. ${ }^{23}$ Success in determining language lateralization in resting-state fMRI is heterogeneous, with the pitfall of showing a more bilateral language distribution. ${ }^{45,46}$ In terms of clinical feasibility, task-based FC offers the advantage of no additional scan time compared with a dedicated resting-state sequence. These reasons support combining standard fMRI activation analysis 
for language lateralization with task-based FC for the network aspect.

\section{Clinical Implications}

Our study suggests that FC may be a potent tool to visualize sideand pathology-dependent alterations of language networks in epilepsy. First, studies showed a correlation between connectivity strength and cognitive function. ${ }^{47}$ In our patient sample, good naming ability was correlated with stronger FC between the right HC and left Wernicke area, while better performance in verbal fluency was seen in patients with less FC in right language areas across all patients. Neuropsychological studies have linked leftsided MTS to worse performance in language testing. ${ }^{3}$ This may imply that patients with left MTS need early special support to reduce language worsening. Because longer disease duration leads to more extensive network changes, earlier surgical treatment could prevent some of these widespread functional network alterations. Furthermore, the results presented here may further help to identify the ideal patients and their optimal treatment strategy at the optimal time point. Improved presurgical imaging of the individual organization of brain functions such as language could ultimately help to better assess the individual risk profile of patients with TLE. Further studies and a longitudinal study design are needed to differentiate whether the observed changes in FC are due to effective plasticity or a mere attempt at compensation for language deficits or whether they are the result of a pathologic process interfering with language function.

\section{Limitations}

Seizure frequency recorded by patients was not considered because its accuracy may be biased due to amnestic seizures. Also, the impact of antiepileptic medication, mood/anxiety, or depression was not investigated in this study. Our selection criteria of low-grade mesiotemporal tumors were strict to keep the group as homogeneous as a tumor cohort could be, however, resulting in a modest sample size of left-sided lesions. While we found significant lesion-specific FC changes compared with the control group, the direct comparison of patient subgroups did not yield significant results. This may be due to the subtlety of alterations not detectable with our modest sample sizes and the statistical necessity of multiple comparison corrections. On the contrary, sample size limitations forced us to combine 2 different language tasks and different numbers of runs, thereby inferring a certain heterogeneity. The rationale behind the inclusion of different numbers of runs was to maximize the time points available for analysis, thus making functional connectivity analysis more robust. ${ }^{24}$ Subsequent studies with larger cohorts may further refine our results.

For both the LI and FC analyses, we used the same ROIs integrated in the CONN toolbox, which are based on an independent component analysis of 497 healthy subjects from the Human Connectome Project dataset. This approach should facilitate the direct comparability between these methods but could, however, potentially "dilute" connectivity measures. Alternatively, we could have used ROIs individually defined by task-based activation maps; this process, however, is prone to bias due to thresholding. Because there is no criterion standard for brain parcellation today, ${ }^{46}$ we are positive that the chosen approach addresses our study aim.

\section{CONCLUSIONS}

Different types of pathologies underlying TLE cause distinct alterations of the functional language connectome. FC analysis identifies these lesion-specific footprints of epileptogenic lesions in a unique fashion. Furthermore, FC strength correlates with clinical language function in patients with TLE. Thus, FC analysis may provide a comprehensive and more global perspective on language processing compared with standard fMRI activation analysis. In summary, these functional imaging findings emphasize the importance of a personalized treatment strategy in patients with TLE.

Disclosures: Lisa Bartha-Doering-UNRELATED: Grants/Grants Pending: Austrian Science Fund, Comments: I am the Principal Investigator of the project FWF KLIF 544 "Prenatal Brain Asymmetry and Language," funded by the Austrian Science Fund, 2016-2020. This project does not interact with the present work. Gregor Kasprian-UNRELATED: Comments: I am the principal investigator of the project FWF 13925-B27 entitled "Epigenetic control of brain wiring role of miRNAs in FASD". This project does not interact with the present work.

\section{REFERENCES}

1. Blumcke I, Spreafico R, Haaker G, et al. Histopathological findings in brain tissue obtained during epilepsy surgery. $N$ Engl J Med 2017;377:1648-56 CrossRef Medline

2. Helmstaedter C. Cognitive outcomes of different surgical approaches in temporal lobe epilepsy. Epileptic Disord 2013;15:221-39 CrossRef Medline

3. Helmstaedter C, Petzold I, Bien CG. The cognitive consequence of resecting nonlesional tissues in epilepsy surgery-results from MRI- and histopathology-negative patients with temporal lobe epilepsy. Epilepsia 2011;52:1402-08 CrossRef Medline

4. Oyegbile TO, Dow C, Jones J, et al. The nature and course of neuropsychological morbidity in chronic temporal lobe epilepsy. Neurology 2004;62:1736-42 CrossRef Medline

5. Bartha-Doering L, Trinka E. The interictal language profile in adult epilepsy. Epilepsia 2014;55:1512-25 CrossRef Medline

6. Austermuehle A, Cocjin J, Reynolds R, et al. Language functional MRI and direct cortical stimulation in epilepsy preoperative planning. Ann Neurol 2017;81:526-37 CrossRef Medline

7. Black DF, Vachha B, Mian A, et al. American Society of Functional Neuroradiology-Recommended fMRI paradigm algorithms for presurgical language assessment. AJNR Am J Neuroradiol 2017;38: E65-73 CrossRef Medline

8. Poeppel D. The neuroanatomic and neurophysiological infrastructure for speech and language. Curr Opin Neurobiol 2014;28:142-49 CrossRef Medline

9. Friston KJ. Functional and effective connectivity: a review. Brain Connect 2011;1:13-36 CrossRef Medline

10. Biswal B, Yetkin FZ, Haughton VM, et al. Functional connectivity in the motor cortex of resting human brain using echo-planar MRI. Magn Reson Med 1995;34:537-41 CrossRef Medline

11. Rosazza C, Ghielmetti F, Minati L, et al. Preoperative language lateralization in temporal lobe epilepsy (TLE) predicts peri-ictal, pre- and post-operative language performance: an fMRI study. Neuroimage Clin 2013;3:73-83 CrossRef Medline

12. Kaestner E, Reyes A, Macari AC, et al. Identifying the neural basis of a language-impaired phenotype of temporal lobe epilepsy. Epilepsia 2019;60:1627-38 CrossRef Medline

13. Louis DN, Ohgaki H, Wiestler OD, et al. The 2007 WHO classification of tumours of the central nervous system. Acta Neuropathol 2007;114:97-109 CrossRef Medline 
14. Louis DN, Perry A, Reifenberger G, et al. The 2016 World Health Organization Classification of Tumors of the Central Nervous System: a summary. Acta Neuropathol 2016;131:803-20 CrossRef Medline

15. Kaplan E, Goodglass H, Weintraub S. Boston Naming Test. Philadelpia: Lea \& Febiger; 1983

16. Aschenbrenner A, Tucha O, Lange K. RWT: Regensburger WortflüssigkeitsTest. Göttingen: Hogrefe; 2000

17. Friston KJ, Holmes AP, Worsley KJ, et al. Statistical parametric maps in functional imaging: a general linear approach. Hum Brain Mapp 1994;2:189-210 CrossRef

18. Wilke M, Lidzba K. LI-tool: a new toolbox to assess lateralization in functional MR-data. J Neurosci Methods 2007;163:128-36 CrossRef Medline

19. Whitfield-Gabrieli S, Nieto-Castanon A. Conn: a functional connectivity toolbox for correlated and anticorrelated brain networks. Brain Connect 2012;2:125-41 CrossRef Medline

20. Seghier ML. Laterality index in functional MRI: methodological issues. Magn Reson Imaging 2008;26:594-601 CrossRef Medline

21. Fair DA, Schlaggar BL, Cohen AL, et al. A method for using blocked and event-related fMRI data to study "resting state" functional connectivity. Neuroimage 2007;35:396-405 CrossRef Medline

22. Behzadi Y, Restom K, Liau J, et al. A component based noise correction method (CompCor) for BOLD and perfusion-based fMRI. Neuroimage 2007;37:90-101 CrossRef Medline

23. Ganger S, Hahn A, Kublbock M, et al. Comparison of continuously acquired resting state and extracted analogues from active tasks. Hum Brain Mapp 2015;36:4053-63 CrossRef Medline

24. Shah LM, Cramer JA, Ferguson MA, et al. Reliability and reproducibility of individual differences in functional connectivity acquired during task and resting state. Brain Behav 2016;6:e00456 CrossRef Medline

25. Davey CE, Grayden DB, Egan GF, et al. Filtering induces correlation in fMRI resting state data. Neuroimage 2013;64:728-40 CrossRef Medline

26. Zalesky A, Fornito A, Bullmore ET. Network-based statistic: identifying differences in brain networks. Neuroimage 2010;53:1197-1207 CrossRef Medline

27. Blumcke I, Thom M, Aronica E, et al. International consensus classification of hippocampal sclerosis in temporal lobe epilepsy: a Task Force Report from the ILAE Commission on Diagnostic Methods. Epilepsia 2013;54:1315-29 CrossRef Medline

28. Bonilha L, Edwards JC, Kinsman SL, et al. Extrahippocampal gray matter loss and hippocampal deafferentation in patients with temporal lobe epilepsy. Epilepsia 2010;51:519-28 CrossRef Medline

29. Coan AC, Campos BM, Yasuda CL, et al. Frequent seizures are associated with a network of gray matter atrophy in temporal lobe epilepsy with or without hippocampal sclerosis. PLoS One 2014;9: e85843 CrossRef Medline

30. Haneef Z, Lenartowicz A, Yeh HJ, et al. Functional connectivity of hippocampal networks in temporal lobe epilepsy. Epilepsia 2014; 55:137-45 CrossRef Medline

31. de Campos BM, Coan AC, Lin Yasuda C, et al. Large-scale brain networks are distinctly affected in right and left mesial temporal lobe epilepsy. Hum Brain Mapp 2016;37:3137-52 CrossRef Medline
32. Covington NV, Duff MC. Expanding the language network: direct contributions from the hippocampus. Trends Cogn Sci 2016;20:86970 CrossRef Medline

33. Vaughan DN, Rayner G, Tailby C, et al. MRI-negative temporal lobe epilepsy: a network disorder of neocortical connectivity. Neurology 2016;87:1934-42 CrossRef Medline

34. Blumcke I, Aronica E, Urbach H, et al. A neuropathology-based approach to epilepsy surgery in brain tumors and proposal for a new terminology use for long-term epilepsy-associated brain tumors. Acta Neuropathol 2014;128:39-54 CrossRef Medline

35. Giulioni M, Marucci G, Martinoni M, et al. Epilepsy associated tumors: review article. World J Clin Cases 2014;2:623-41 CrossRef Medline

36. Noll KR, Sullaway C, Ziu M, et al. Relationships between tumor grade and neurocognitive functioning in patients with glioma of the left temporal lobe prior to surgical resection. Neuro Oncol 2015;17:580-87 CrossRef Medline

37. Briganti C, Sestieri C, Mattei PA, et al. Reorganization of functional connectivity of the language network in patients with brain gliomas. AJNR Am J Neuroradiol 2012;33:1983-90 CrossRef Medline

38. Ghinda CD, Duffau H. Network plasticity and intraoperative mapping for personalized multimodal management of diffuse lowgrade gliomas. Front Surg 2017;4:3 CrossRef Medline

39. Muhlhofer W, Tan YL, Mueller SG, et al. MRI-negative temporal lobe epilepsy: what do we know? Epilepsia 2017;58:727-42 CrossRef Medline

40. Weber B, Wellmer J, Reuber M, et al. Left hippocampal pathology is associated with atypical language lateralization in patients with focal epilepsy. Brain 2006;129:346-51 CrossRef Medline

41. Mazoyer B, Zago L, Jobard G, et al. Gaussian mixture modeling of hemispheric lateralization for language in a large sample of healthy individuals balanced for handedness. PLoS One 2014;9: e101165 CrossRef Medline

42. Pravata E, Sestieri C, Mantini D, et al. Functional connectivity MR imaging of the language network in patients with drug-resistant epilepsy. AJNR Am J Neuroradiol 2011;32:532-40 CrossRef Medline

43. Briellmann RS, Labate A, Harvey AS, et al. Is language lateralization in temporal lobe epilepsy patients related to the nature of the epileptogenic lesion? Epilepsia 2006;47:916-20 CrossRef Medline

44. Fox MD, Raichle ME. Spontaneous fluctuations in brain activity observed with functional magnetic resonance imaging. Nat Rev Neurosci 2007;8:700-11 CrossRef Medline

45. Teghipco A, Hussain A, Tivarus ME. Disrupted functional connectivity affects resting state based language lateralization. Neuroimage Clin 2016;12:910-27 CrossRef Medline

46. Doucet GE, Pustina D, Skidmore C, et al. Resting-state functional connectivity predicts the strength of hemispheric lateralization for language processing in temporal lobe epilepsy and normals. Hum Brain Mapp 2015;36:288-303 CrossRef Medline

47. Trimmel K, van Graan AL, Caciagli L, et al. Left temporal lobe language network connectivity in temporal lobe epilepsy. Brain 2018 141:2406-18 CrossRef Medline 